\title{
RADIO EMISSION FROM THE MOON AND THE NATURE OF ITS SURFAGE
}

\author{
V. S. TROITZKY AND S. E. KHAIKIN \\ Gorky State University and Physical Institute of the Academy of Sciences, \\ Moscow, U.S.S.R.
}

A theoretical study of the integral radio emission of the moon, measured at the wave-length of $3.2 \mathrm{~cm}$. (Zelinskaja and Troitzky[1]; Kajdanovsky, Turusbekov and Khaikin[2]), was carried out at the Gorky radio astronomical station 'Zimenky' and at the Physical Institute of the Academy of Sciences of the U.S.S.R. The following expression for the average radio temperature of the entire lunar disk, as a function of the lunar phase, $\Omega t$, was obtained (Troitzky, 1954)[3]:

$$
T_{c}(t)=0.92\left(\mathrm{I}-k_{0}\right)\left(T_{n}+0 \cdot 344 \Theta\right)+\frac{0 \cdot 37\left(\mathrm{I}-k_{0}\right) \Theta}{\sqrt{\mathrm{I}+2 \delta+2 \delta^{2}}} \cos (\Omega t-\xi) .
$$

Here $\tan \xi=\delta /(\mathrm{I}+\delta)$ and $\delta=\beta / \kappa$, where $\beta$ is the attenuation coefficient of the thermal wave, $\kappa$ the power attenuation coefficient of the radio wave. Further, $T_{m}=374^{\circ} \mathrm{K}$. is the temperature of the subsolar point, $T_{n}$ is the temperature at the lunar midnight, $\Theta=T_{m}-T_{n}$ and $k_{0}$ is the reflexion coefficient of radio waves for vertical incidence $\left(k_{0} \approx 0 \cdot \mathrm{I}\right)$. The numerical coefficients in equation ( $I$ ) were obtained as a result of averaging the Fresnel reflexion coefficients over the whole disk. The degree of polarization of the total radio emission was calculated and was found to be about $4 \%$.

Two cycles of measurements of the radio emission from the moon at the wave-length of $3.2 \mathrm{~cm}$., carried out in 1952 (Troitzky and Zelinskaja) [4] and 1955, yielded the result that the mean radio temperature, determined with a precision of \pm 5 to $\pm 7 \%$, does not depend upon phase. The constant component of the radio temperature was found to be:

$$
T_{c}=170^{\circ} \pm 10^{\circ} \mathrm{K} .
$$

From this result combined with equation (I) the temperature $T_{n}$ at the lunar midnight was found to be $115^{\circ} \mathrm{K}$. The amplitude of the variable 406 
part of the radio temperature may be estimated from the maximum displacement of the 'centre of gravity' of the radiation relative to the geometric centre of the moon. Measurements show that no systematic displacement of the centre of emission as a function of phase exists up to \pm 0.5 minutes of arc. The amplitude of the variation of the lunar radio temperature is consequently less than $10^{\circ} \mathrm{K}$.

The experimental data of the radio emission from the moon obtained on $3.2 \mathrm{~cm}$. and the data given by Piddington and Minnett (1949) [5] for $\mathrm{I} \cdot 25 \mathrm{~cm}$. were discussed on the basis of equation (I). The reduction shows that neither the one-layer model of the surface structure nor the doublelayer model contradict the results obtained on these two wave-lengths. It was shown, however, that the value for the mean radio temperature of the moon obtained by Piddington and Minnett contradicts optical temperature measurements and the thermal properties of the lunar rocks (Troitzky[6]). Assuming the value $(k \rho c)^{-1 / 2}$ as known from optical measurements (thermal conductivity $k=2.5 \times 10^{-6}$, density $\rho=2$, thermal capacity $c=0 \cdot 2$ ) the electrical conductivity of the lunar rocks was found to be $\sigma_{3}=5.4 \times 10^{8} \mathrm{cgs}$. (penetration depth $=8.7 \mathrm{~cm}$.) for $\lambda=3.2 \mathrm{~cm}$. and $\sigma_{1}=\mathrm{I} \cdot 4 \times \mathrm{IO}^{9} \mathrm{cgs}$. (penetration depth $=3.3 \mathrm{~cm}$.) for $\lambda=\mathrm{I} \cdot 25 \mathrm{~cm}$. The comparatively high values of the electric conductivity obtained suggest that the content of calcium, natrium and iron oxides in the lunar surface material is considerable. This conclusion agrees with the results of investigations carried out in reflected light (Budnikova, 1953 [7]; Borissova, I953 $\left.{ }^{[8]}\right)$.

In order to solve the problem of the structure of the lunar surface, further precise measurements of the radio emission of the moon, particularly on 8 and $4 \mathrm{~mm}$. waves, are needed.

\section{REFERENGES}

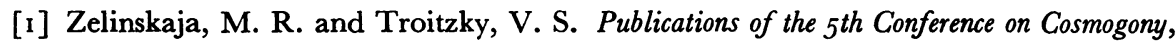
p. 99,1956 .

[2] Kajdanovsky, N. L., Turusbekov, M. T. and Khaikin, S. E. Ibid. p. 347, 1956.

[3] Troitzky, V. S. A.J. U.S.S.R. 31, 6, 51 I , 1954 .

[4] Troitzky, V. S. and Zelinskaja, M. R. A.J. U.S.S.R. (in print).

[5] Piddington, J. H. and Minnett, H. G. Aust. J. Sci. Res. A, 2, 63, 1949.

[6] Troitzky, V. S. Publications of the 5th Conference on Cosmogony, p. 325, 1956.

[7] Budnikova, N. A. Bulletin of the Leningrad University, Math. Phys. Chem. Series no. 8, $71,1953$.

[8] Borissova, A. N. Ibid. series no. 8, 89, 1953. 